\title{
Switching Rate in Selective Cooperative Relaying
}

\author{
Diomidis S. Michalopoulos*, Athanasios S. Lioumpas ${ }^{\dagger}$, George K. Karagiannidis ${ }^{\dagger}$, and Robert Schober* \\ * Department of Electrical \& Computer Engineering, The University of British Columbia, Vancouver, BC, V6T 1Z4, Canada \\ E-mail: $\{$ dio, rschober\}@ece.ubc.ca \\ ${ }^{\dagger}$ Department of Electrical \& Computer Engineering, Aristotle University of Thessaloniki, GR-54124, Thessaloniki, Greece \\ E-mail:\{alioumpa, geokarag\}@auth.gr
}

\begin{abstract}
In selective cooperative relaying only a single relay out of the set of available relays is activated at a time, hence the available power and bandwidth resources are efficiently utilized. However, implementing selective cooperative relaying in time-varying channels may cause frequent relay switchings that deteriorate the overall performance. In this paper, we study the rate at which a relay switching occurs in selective cooperative relaying applications with two available relays, operating over time-varying fading channels. In particular, we derive closedform expressions for the relay switching rate (measured in $\mathbf{H z}$ ) of opportunistic relaying (OR) and distributed switch and stay combining (DSSC). Numerical results manifest that DSSC yields a considerably lower relay switching rate than $O R$.
\end{abstract}

\section{INTRODUCTION}

Cooperative relaying has been recently proposed as a means of achieving the beneficial effects of diversity in wireless communications systems, without employing multiple antennas at neither the receiver nor the transmitter. The most common cooperative relaying protocols were introduced in [1]; however, they consider the scenario where only a single relay is available for cooperation. In cases where multiple relaying terminals are utilized, an effective means of achieving diversity through cooperating terminals is activating only a single relay at a time so as to limit the spectral inefficiency effects of multiple orthogonal transmissions.

Previous works on single relay selection in cooperative relaying scenarios include [2]- [5], where the relay selection was based on a maximum signal-to-noise-ratio (SNR) policy. Due to the somewhat opportunistic usage of the available resources, the relay selection protocol based upon the maximum SNR rule is termed opportunistic relaying (OR). A simpler alternative to OR, for the case of two available relaying terminals, is the so-called distributed switch and stay combining (DSSC) protocol proposed in [6]. According to DSSC, a single relay remains active for as long as the corresponding SNR is greater than a predetermined threshold value; should this condition be violated, a relay switching occurs. The DSSC protocol hence requires only a single end-to-end channel estimation for each transmission, reducing thus the overall complexity while achieving the same outage performance as OR, albeit inferior error performance [6]. In the sequel, we use the term "selective cooperative relaying" to refer to the OR and DSSC protocols, i.e., to protocols where single relay selection takes place.

Despite the above benefits of selective cooperative relaying, however, a major issue that needs to be addressed is the rate at which a switching of the active relaying terminal occurs in practical scenarios where the fading in each of the links involved is time-varying. In fact, this rate reflects the number of times per second the system has to switch from one relaying terminal to another, and corresponds to a complexity measure regarding the implementation of selective cooperative relaying in practice. More specifically, frequent relay switchings may cause synchronization problems due to the fact that the system needs to repeat the initialization process each time the active relay changes, in order to readapt to the channel conditions of the new branch. To the best of the authors' knowledge, the concept of relay switchings in time-varying fading environments has not been addressed in the literature so far.

In this paper, we study the effect of the time-varying nature of fading channels in selective cooperative relaying applications with two available relaying terminals. In particular, we provide closed-form expressions for the relay switching rate (measured in $\mathrm{Hz}$ ) of OR and DSSC, defined as the number of times per second that a switching of the active relay takes place; that is, the destination stops receiving from a certain relaying terminal and connects to another one. The derived expressions are functions of the average channel gains and the maximum Doppler frequency of each of the source-relay and relay-destination links involved. Moreover, these expressions consider the case of independent but not necessarily identically distributed (i.n.i.d.) Rayleigh fading channels, and account for both amplify-and-forward (AF) and decode-and-forward (DF) relaying. A set of numerical examples is provided showing that DSSC results in a considerably lower relay switching rate than OR.

\section{BACKGROUND}

We consider the cooperative relaying setup where a source terminal, $S$, communicates with a destination terminal, $D$, with the aid of $L$ relaying terminals which are denoted here by $R_{i}, i \in\{1, \ldots, L\}$; the relays are assumed to be half-duplex, and may operate in either the DF or the AF mode. In this paper, we are interested in the case of $L=2$ available relays. We adopt the general notation, $a_{A B}$, to denote the channel gain of the link between terminals $A$ and $B$, so that the channel gain of, e.g., the $S-R_{i}$ link, is represented by $a_{S R_{i}}$. The fading in each of the links involved is assumed to be Rayleigh distributed, with probability density function (PDF) given by $f_{a_{A B}}(x)=\left(2 x / \Omega_{A B}\right) \exp \left(-x^{2} / \Omega_{A B}\right)$, where $\Omega_{A B}$ 
represents the average squared channel gain of the $A-B$ link, e.g., $\Omega_{S R_{i}}=E\left[a_{S R_{i}}^{2}\right]$, with $E[\cdot]$ denoting expectation. We denote the maximum Doppler frequency of the $A-B$ link by $\mathcal{F}_{A B}$, e.g., the maximum Doppler frequency of the $S$ - $R_{i}$ link is denoted by $\mathcal{F}_{S R_{i}}$. Moreover, all terminals are assumed to transmit with identical power, $P_{T}$, while the noise power in all of the links involved is identical and denoted by $N_{0}$.

Throughout this work, two selective cooperative relaying protocols are considered: The opportunistic relaying (OR) protocol presented in [3], and a variant of the two-relay distributed switch and stay combining (DSSC) protocol proposed in [6], which is referred to as DSSC-B here. Next, the modes of operation of OR and DSSC-B are given in detail.

\section{A. Opportunistic Relaying (OR)}

The OR protocol consists of selecting a single relay out of the set of $L$ available relays. In order to perform selection, the equivalent channel gain of the $i$ th end-to-end path is defined as

$$
a_{i}=\min \left(a_{S R_{i}}, a_{R_{i} D}\right) .
$$

Note that the above definition accounts for determining the end-to-end path based on the weakest intermediate link. It is appropriate for DF relaying because it corresponds to an outage-equivalent of the end-to-end DF channel, since the outage probability of DF relaying equals the cumulative distribution function (CDF) of $a_{i}$ evaluated at the outage threshold SNR. Additionally, it provides a tight upper bound on the end-to-end SNR for AF relaying [7]. Therefore, in the sequel it is assumed that the activated relay, $R_{b}$, is selected according to

$$
b=\arg \max _{i \in\{1, \ldots, L\}}\left(a_{i}\right) .
$$

\section{B. $D S S C-B$}

We refer to the considered DSSC scheme as DSSC-B, since it varies from DSSC in exactly the same way as SSC-B varies from SSC-A in [8]. In brief, according to SSC-A a branch switching takes place whenever the SNR of the active branch is lower than a threshold value; according to SSC-B, a switching occurs whenever the SNR of the active branch down-crosses the threshold. In that sense, if the SNR of the active branch in SSC-B still remains below the threshold after the switching, the system stays connected to that branch until a down-crossing occurs.

The reasoning behind studying DSSC-B instead of the original DSSC protocol presented in [6] lies in the fact that DSSC-B yields less frequent relay switchings than DSSC, similarly as SSC-B yields less frequent switchings than SSCA [8]. Hence, in order to avoid excessive switchings that may take place when both the available end-to-end channels are not strong enough, a switching occurs if $a_{b}<\sqrt{T / \Gamma}$, where $\Gamma=P_{T} / N_{0}$ denotes the ratio of the power transmitted by the source and each relay divided by the noise power, i.e., the common SNR without fading; in other words, a switching occurs whenever the SNR of the active branch down-crosses the switching threshold, $T$; the system then stays connected with the new branch, regardless of whether the SNR of the new branch is greater or lower than $T$, until this SNR downcrosses $T$. The simplicity of DSSC-B over OR lies in the fact that in each training period, only a single end-to-end channel has to be estimated.

Let us denote by $a_{i}^{j}$ and $R_{b}^{j}$ the equivalent end-to-end channel of the $j$ th branch and the active relay, respectively, for a transmission period $j$. If $R_{b}^{j}=R_{1}, R_{b}^{j+1}$ is determined by

$$
R_{b}^{j+1}=\left\{\begin{array}{l}
R_{1} \text { if }\left[a_{1}^{j-1}<\sqrt{T / \Gamma} \text { or } a_{1}^{j}>\sqrt{T / \Gamma}\right] \\
R_{2} \text { if }\left[a_{1}^{j-1}>\sqrt{T / \Gamma} \text { and } a_{1}^{j}<\sqrt{T / \Gamma}\right]
\end{array}\right.
$$

Equivalently, if $R_{b}^{j}=R_{2}, R_{b}^{j+1}$ is determined by

$$
R_{b}^{j+1}=\left\{\begin{array}{l}
R_{2} \text { if }\left[a_{2}^{j-1}<\sqrt{T / \Gamma} \text { or } a_{2}^{j}>\sqrt{T / \Gamma}\right] \\
R_{1} \text { if }\left[a_{2}^{j-1}>\sqrt{T / \Gamma} \text { and } a_{2}^{j}<\sqrt{T / \Gamma}\right]
\end{array} .\right.
$$

It is interesting to note that the end-to-end path between source and destination in both OR and DSSC-B is treated as a virtual channel with channel gain $a_{i}=\min \left(a_{S R_{i}}, a_{R_{i} D}\right)$. The OR scheme can be thus regarded as a virtual selection diversity scheme, where the instantaneous channel gain of the $i$ th input branch is $a_{i}$; the DSSC-B scheme can be interpreted as a virtual SSC-B scheme with channel gains $a_{1}$ and $a_{2}$. The selection is performed at the destination terminal, which collects the channel state information (CSI) of all the links involved. Then, after determining the "best" relay, the destination sends a feedback message to the relays indicating the activation of the selected relay and the deactivation of the previously selected relay.

\section{Relay SWitching Rate OF OR}

Theorem 1: The relay switching rate of OR with two available relays and i.n.i.d. fading is given by

$$
\begin{aligned}
& S R_{O R}=\frac{\pi \sqrt{2 \Omega_{1} \Omega_{2}} \Omega_{S R_{1}} \Omega_{S R_{2}} \sqrt{\Omega_{R_{1} D} \mathcal{F}_{R_{1} D}^{2}+\Omega_{R_{2} D} \mathcal{F}_{R_{2} D}^{2}}}{\left(\Omega_{1}+\Omega_{2}\right)^{3 / 2}\left(\Omega_{S R_{1}}+\Omega_{R_{1} D}\right)\left(\Omega_{S R_{2}}+\Omega_{R_{2} D}\right)} \\
&+ \frac{\pi \sqrt{2 \Omega_{1} \Omega_{2}} \Omega_{R_{1} D} \Omega_{S R_{2}} \sqrt{\Omega_{S R_{1}} \mathcal{F}_{S R_{1}}^{2}+\Omega_{R_{2} D} \mathcal{F}_{R_{2} D}^{2}}}{\left(\Omega_{1}+\Omega_{2}\right)^{3 / 2}\left(\Omega_{S R_{1}}+\Omega_{R_{1} D}\right)\left(\Omega_{S R_{2}}+\Omega_{R_{2} D}\right)} \\
&+ \frac{\pi \sqrt{2 \Omega_{1} \Omega_{2}} \Omega_{R_{2} D} \Omega_{S R_{1}} \sqrt{\Omega_{S R_{2}} \mathcal{F}_{S R_{2}}^{2}+\Omega_{R_{1} D} \mathcal{F}_{R_{1} D}^{2}}}{\left(\Omega_{1}+\Omega_{2}\right)^{3 / 2}\left(\Omega_{S R_{1}}+\Omega_{R_{1} D}\right)\left(\Omega_{S R_{2}}+\Omega_{R_{2} D}\right)} \\
&+\frac{\pi \sqrt{2 \Omega_{1} \Omega_{2}} \Omega_{R_{1} D} \Omega_{R_{2} D} \sqrt{\Omega_{S R_{1}} \mathcal{F}_{S R_{1}}^{2}+\Omega_{S R_{2}} \mathcal{F}_{S R_{2}}^{2}}}{\left(\Omega_{1}+\Omega_{2}\right)^{3 / 2}\left(\Omega_{S R_{1}}+\Omega_{R_{1} D}\right)\left(\Omega_{S R_{2}}+\Omega_{R_{2} D}\right)}
\end{aligned}
$$

where

$$
\Omega_{i}=E\left[a_{i}^{2}\right]=\frac{\Omega_{S R_{i}} \Omega_{R_{i} D}}{\Omega_{S R_{i}}+\Omega_{R_{i} D}}
$$

represents the average squared value of $a_{i}, i \in\{1,2\}$.

Proof: Let us consider the random processes

$$
a_{1}(t)=\min \left(a_{S R_{1}}(t), a_{R_{1} D}(t)\right)
$$




$$
a_{2}(t)=\min \left(a_{S R_{2}}(t), a_{R_{2} D}(t)\right)
$$

which correspond to the fading processes of the virtual end-toend channels $S-R_{1}-D$ and $S-R_{2}-D$, respectively. Additionally, let us define the random process $Z(t)$ as

$$
Z(t)=a_{1}(t)-a_{2}(t)
$$

so that the active relay in each time instance $t$ is determined by the signum of $Z(t)$ in this time instance, i.e., $R_{1}$ is active at time $t$ if $Z(t)>0 ; R_{2}$ is active at time $t$ if $Z(t)<0$. Consequently, in order to derive the average relay switching rate it suffices to evaluate the average number of times the process $Z(t)$ crosses zero. This is equivalent to obtaining the level crossing rate (LCR) of $Z(t)$, evaluated at zero. Then, the positive-going LCR of $Z(t)$ corresponds to the average number of times the system switches from $R_{2}$ to $R_{1}$, while the negative-going LCR of $Z(t)$ accounts for the average number of times the system switches from $R_{1}$ to $R_{2}$.

The relay switching rate equals the sum of positive-going and negative-going zero-crossing rates of $Z(t)$, which can be expressed as [9]

$$
S R_{O R}=\int_{-\infty}^{0}|\dot{z}| f(0, \dot{z}) d \dot{z}+\int_{0}^{\infty} \dot{z} f(0, \dot{z}) d \dot{z}
$$

where $f(z, \dot{z})$ denotes the joint PDF of $Z(t)$ and the timederivative of $Z(t), Z(t)$. Because of the independence of the fading process and its time derivative, in each of the intermediate links involved, owing to the Rayleigh fading assumption, the processes $Z(t)$ and $Z(t)$ are independent [10]. Therefore, $f(z, \dot{z})$ can be expressed as $f(z, \dot{z})=f_{Z}(z) f_{\dot{Z}}(\dot{z})$, with $f_{Z}(\cdot)$ and $f_{\dot{Z}}(\cdot)$ denoting the PDFs of $Z(t)$ and $\dot{Z}(t)$, respectively. Equation (10) thus yields

$$
\begin{aligned}
S R_{O R} & =f_{Z}(0)\left[\int_{-\infty}^{0}|\dot{z}| f_{\dot{Z}}(\dot{z}) d \dot{z}+\int_{0}^{\infty} \dot{z} f_{\dot{Z}}(\dot{z}) d \dot{z}\right] \\
& =2 f_{Z}(0) \int_{0}^{\infty} \dot{z} f_{\dot{Z}}(\dot{z}) d \dot{z}
\end{aligned}
$$

where we used the fact that the two integrals in (11a) are equal to each other since, apparently, the number of times the system switches from $R_{1}$ to $R_{2}$ equals that of switching from $R_{2}$ to $R_{1}$, in the long run. The PDF of $Z(t)$ evaluated at the origin is derived as (see Appendix A)

$$
f_{Z}(0)=\frac{\sqrt{\pi} \sqrt{\Omega_{1} \Omega_{2}}}{\left(\Omega_{1}+\Omega_{2}\right)^{3 / 2}} .
$$

For the second term in (11) we obtain (see Appendix B)

$$
\begin{aligned}
& \int_{0}^{\infty} \dot{z} f_{\dot{z}}(\dot{z}) d \dot{z}=\frac{\sqrt{\pi} \Omega_{1} \Omega_{2} \sqrt{\Omega_{R_{1} D} \mathcal{F}_{R_{1} D}^{2}+\Omega_{R_{2} D} \mathcal{F}_{R_{2} D}^{2}}}{\sqrt{2} \Omega_{R_{1} D} \Omega_{R_{2} D}} \\
& +\frac{\sqrt{\pi} \Omega_{1} \Omega_{2} \sqrt{\Omega_{S R_{1}} \mathcal{F}_{S R_{1}}^{2}+\Omega_{R_{2} D} \mathcal{F}_{R_{2} D}^{2}}}{\sqrt{2} \Omega_{S R_{1}} \Omega_{R_{2} D}} \\
& +\frac{\sqrt{\pi} \Omega_{1} \Omega_{2} \sqrt{\Omega_{S R_{2}} \mathcal{F}_{S R_{2}}^{2}+\Omega_{R_{1} D} \mathcal{F}_{R_{1} D}^{2}}}{\sqrt{2} \Omega_{R_{1} D} \Omega_{S R_{2}}} \\
& +\frac{\sqrt{\pi} \Omega_{1} \Omega_{2} \sqrt{\Omega_{S R_{1}} \mathcal{F}_{S R_{1}}^{2}+\Omega_{S R_{2}} \mathcal{F}_{S R_{2}}^{2}}}{\sqrt{2} \Omega_{S R_{1}} \Omega_{S R_{2}}}
\end{aligned}
$$

The relay switching rate of OR for the case of two relays with i.n.i.d. fading channels is obtained by substituting (12) and (13) into (11b), completing the proof.

\section{RElay SWITChing Rate OF DSSC-B}

Theorem 2: The relay switching rate for the two-relay DSSC-B setup is derived as shown in (14) at the top of the next page.

Proof: In the two-relay DSSC-B scheme, a relay switching occurs whenever the negative-going slope of the output SNR crosses the switching threshold, $T$; equivalently, a relay switching occurs whenever the process $a_{i}(t), i \in\{1,2\}$, crosses $\sqrt{T / \Gamma}$ in a negative-going direction; recall that $\Gamma$ denotes the common SNR without fading. The switching rate for relay $R_{i}, i \in\{1,2\}$, is given by

$$
N_{i}(T)=\int_{-\infty}^{0}|x| f_{a_{i}, \dot{a}_{i}}(\sqrt{T / \Gamma}, x) d x
$$

where $f_{a_{i}, \dot{a}_{i}}(\cdot, \cdot)$ denotes the joint PDF of $a_{i}(t)$ and $\dot{a}_{i}(t)$. Therefore, using the fact that $a_{i}(t)$ and $\dot{a}_{i}(t)$ are independent processes, (15) yields

$$
N_{i}(T)=\sqrt{2 \pi}\left(\sqrt{\frac{T \mathcal{F}_{S R_{i}}^{2}}{\Gamma \Omega_{S R_{i}}}}+\sqrt{\frac{T \mathcal{F}_{R_{i} D}^{2}}{\Gamma \Omega_{R_{i} D}}}\right) e^{-\frac{\Omega_{S R_{i}}+\Omega_{R_{i} D}}{\Omega_{S R_{i} \Omega_{i} D}} \frac{T}{\Gamma}} .
$$

The steady-state probability, $\rho_{i}^{D S S C}(T)$, of activating $R_{i}$ in the DSSC-B scheme is derived in Appendix C as shown in (17) and (18) at the top of the next page, where $F_{a_{i}^{2}}(x)=1-$ $\exp \left(-x / \Omega_{i}\right)$ denotes the CDF of $a_{i}^{2}(t), i \in\{1,2\}$. Therefore, the relay switching rate can be expressed as

$$
S R_{D S S C}(T)=\rho_{1}^{D S S C}(T) N_{1}(T)+\rho_{2}^{D S S C}(T) N_{2}(T) .
$$

Hence, after some algebraic manipulations, we arrive at (14).

\section{NUMERICAL EXAMPLES AND DiscusSiON}

\section{A. Implementation Issues}

In classical diversity communication systems (e.g. conventional selection combining or switch and stay combining, where no relaying takes place), the switching between the 


$$
\begin{aligned}
S R_{D S S C}(T)= & \frac{\sqrt{2 \pi} e^{-\frac{T\left(\Omega_{1}+\Omega_{2}\right)}{\Gamma \Omega_{1} \Omega_{2}}}\left[\left(e^{\frac{2 T}{\Gamma \Omega_{2}}}-e^{\frac{T}{\Gamma \Omega_{2}}}\right)\left(\sqrt{\frac{T \mathcal{F}_{S R_{1}}^{2}}{\Gamma \Omega_{S R_{1}}}}+\sqrt{\frac{T \mathcal{F}_{R_{1} D}^{2}}{\Gamma \Omega_{R_{1} D} D}}\right)+\left(e^{\frac{2 T}{\Gamma \Omega_{1}}}-e^{\frac{T}{\Gamma \Omega_{1}}}\right)\left(\sqrt{\frac{T \mathcal{F}_{S R_{2}}^{2}}{\Gamma \Omega_{S}}}+\sqrt{\frac{T \mathcal{F}_{R_{2} D}^{2}}{\Gamma R_{2} D}}\right)\right]}{2 e^{\frac{T}{\Gamma \Omega_{1}}}-e^{\frac{2 T}{\Gamma \Omega_{1}}}+2 e^{\frac{T}{\Gamma \Omega_{2}}}-e^{\frac{2 T}{\Gamma \Omega_{2}}}-2 e^{\frac{T\left(\Omega_{1}+\Omega_{2}\right)}{\Gamma \Omega_{1} \Omega_{2}}}+e^{\frac{T\left(\Omega_{1}+2 \Omega_{2}\right)}{\Gamma \Omega_{1} \Omega_{2}}}+e^{\frac{T\left(2 \Omega_{1}+\Omega_{2}\right)}{\Gamma \Omega_{1} \Omega_{2}}}-2} \\
& +\frac{\sqrt{2 \pi}\left(\sqrt{\frac{T \mathcal{F}_{S R_{1}}^{2}}{\Gamma \Omega_{S R_{1}}}}+\sqrt{\frac{T \mathcal{F}_{R_{1} D}^{2}}{\Gamma \Omega_{R_{1} D}}}+\sqrt{\frac{T \mathcal{F}_{S R_{2}}^{2}}{\Gamma \Omega_{S R_{2}}}}+\sqrt{\frac{T \mathcal{F}_{R_{2} D}^{2}}{\Gamma \Omega_{R_{2} D}}}\right)\left(e^{\frac{T}{\Gamma \Omega_{1}}}-1\right)\left(e^{\frac{T}{\Gamma \Omega_{2}}}-1\right)}{2 e^{\frac{T}{\Gamma \Omega_{1}}}-e^{\frac{2 T}{\Gamma \Omega_{1}}}+2 e^{\frac{T}{\Gamma \Omega_{2}}}-e^{\frac{2 T}{\Gamma \Omega_{2}}}-2 e^{\frac{T\left(\Omega_{1}+\Omega_{2}\right)}{\Gamma \Omega_{1} \Omega_{2}}}+e^{\frac{T\left(\Omega_{1}+2 \Omega_{2}\right)}{\Gamma \Omega_{1} \Omega_{2}}}+e^{\frac{T\left(2 \Omega_{1}+\Omega_{2}\right)}{\Gamma \Omega_{1} \Omega_{2}}}-2}
\end{aligned}
$$

$$
\begin{aligned}
\rho_{1}^{D S S C}(T) & =\frac{F_{a_{2}^{2}}(T / \Gamma)\left[F_{a_{2}^{2}}(T / \Gamma)-1\right]\left[1-F_{a_{1}^{2}}(T / \Gamma)+F_{a_{1}^{2}}^{2}(T / \Gamma)\right]}{F_{a_{1}^{2}}(T / \Gamma)\left[F_{a_{1}^{2}}(T / \Gamma)-1\right]\left[1-2 F_{a_{2}^{2}}(T / \Gamma)+2 F_{a_{2}^{2}}^{2}(T / \Gamma)\right]+F_{a_{2}^{2}}(T / \Gamma)\left[F_{a_{2}^{2}}(T / \Gamma)-1\right]} \\
\rho_{2}^{D S S C}(T) & =\frac{F_{a_{1}^{2}}(T / \Gamma)\left[F_{a_{1}^{2}}(T / \Gamma)-1\right]\left[1-F_{a_{2}^{2}}(T / \Gamma)+F_{a_{2}^{2}}^{2}(T / \Gamma)\right]}{F_{a_{2}^{2}}(T / \Gamma)\left[F_{a_{2}^{2}}(T / \Gamma)-1\right]\left[1-2 F_{a_{1}^{2}}(T / \Gamma)+2 F_{a_{1}^{2}}^{2}(T / \Gamma)\right]+F_{a_{1}^{2}}(T / \Gamma)\left[F_{a_{1}^{2}}(T / \Gamma)-1\right]}
\end{aligned}
$$

diversity branches causes several problems, such as "an internal outage" due to the corruption of the receiver filters and data signal chains, as well as phase estimation failures [10]. In cooperative relaying systems, however, where signals have to be exchanged between spatially distributed nodes, an additional important issue has to be addressed; such issue is time synchronization, which requires the local clocks of the relay nodes to be synchronized, requiring various degrees of precision [11].

Particularly in cooperative relaying systems employing mobile relays, the time delay between the transmitter and the destination may change dramatically as soon as a relay switching occurs. Time synchronization is then particularly challenging since the network dynamics such as propagation time or physical channel access time are in general nondeterministic, because of the relative distances between source, relays, and destination. Therefore, it is evident that frequent relay switchings are not desirable as they significantly increase the implementation complexity of relay selection protocols, rendering them difficult to implement in fast fading scenarios.

\section{B. Numerical Results}

In this subsection, we illustrate some numerical results, regarding the relay switching rate of both OR and DSSC-B. All the results shown in this section were also confirmed by simulations. As already mentioned, the relay switching rate constitutes a vital part in the design and implementation of selective cooperative relaying, due to the complexity issues that each relay switching entails.

Fig. 1 depicts the normalized switching rates of both OR and DSSC-B versus the ratio $\mathcal{F}_{S R_{i}} / \mathcal{F}_{R_{i} D}$ for $L=2$ relays, when the fading powers in the $S-R$ and $R-D$ links are unbalanced. Specifically, the average channel gains of the $S-R_{1}$ and $S-R_{2}$ links are assumed equal to each other, and the same is true for the $R_{1}-D$ and $R_{2}-D$ links; the difference in the corresponding average SNRs is $10 \mathrm{~dB}$, so that $\Gamma \Omega_{S R_{i}}=\Gamma \Omega_{R_{i} D} \pm 10 \mathrm{~dB}$, $i \in\{1,2\}$. As observed from Fig. 1, the switching rate of DSSC-B is always smaller than that of OR, with the latter being under specific conditions even ten times greater than the former. This verifies the intuition that DSSC-B leads to less frequent relay switchings, accounting thus for simpler practical implementation. It should be noted that in Fig. 1 the switching threshold, $T$, is that threshold which leads to the maximum possible switching rate of DSSC-B, which is determined through numerical optimization methods. In other words, the DSSC-B curves depict the worst case in terms of switching rate, demonstrating that even in this scenario the DSSC-B switching rate is much smaller than that of OR.

In Fig. 2, the switching rates of OR and DSSC-B are plotted for $L=2$ relays and several values of the switching threshold, $T$, assuming that the channel gains in the $S-R$ and $R-D$ links are unbalanced, as well as that $\mathcal{F}_{S R_{i}}=2 \mathcal{F}_{R_{i} D}, i \in\{1,2\}$. For each of the scenarios shown here, the switching rate of DSSC-B is considerably lower than that of OR. The ratio of switching rates is, however, crucially dependent on the switching threshold of DSSC-B, implying that the difference of the switching rates of OR and DSSC-B shown in Fig. 1 is significantly expanded for $T$ values different from the worst case (in terms of relay switching rate) for DSSC-B.

\section{CONCLUSION}

We conducted a study of selective cooperative relaying with two available relays in time-varying fading channels. In particular, we derived the relay switching rate for selective cooperative relaying, which reflects the concept of how frequently a cooperative scheme switches from one relay to another. Numerical results indicated that selecting the active relay in a maximum SNR-based fashion results in a considerably higher switching rate than selecting the relay in a DSSC-B fashion. Therefore, considering the complexity issues associated with frequent relay switching, DSSC-B leads to a 


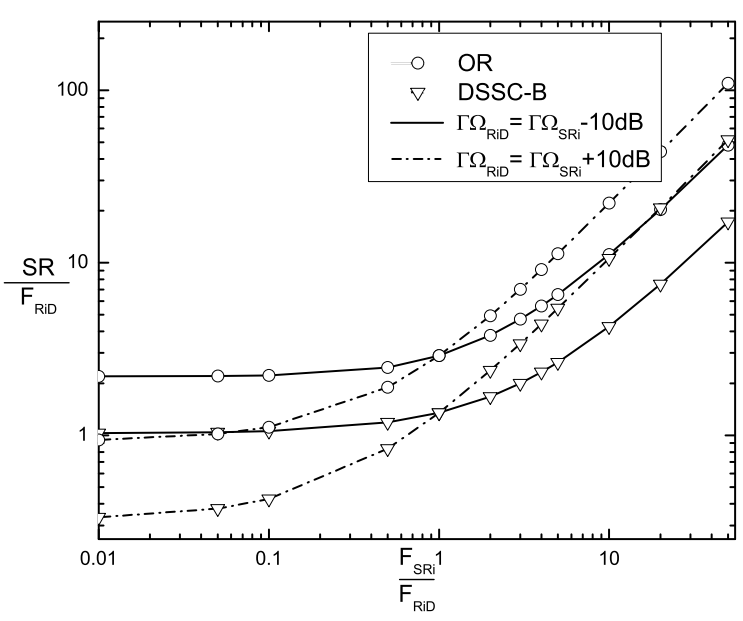

Fig. 1. Relay switching rates of OR and DSSC-B.

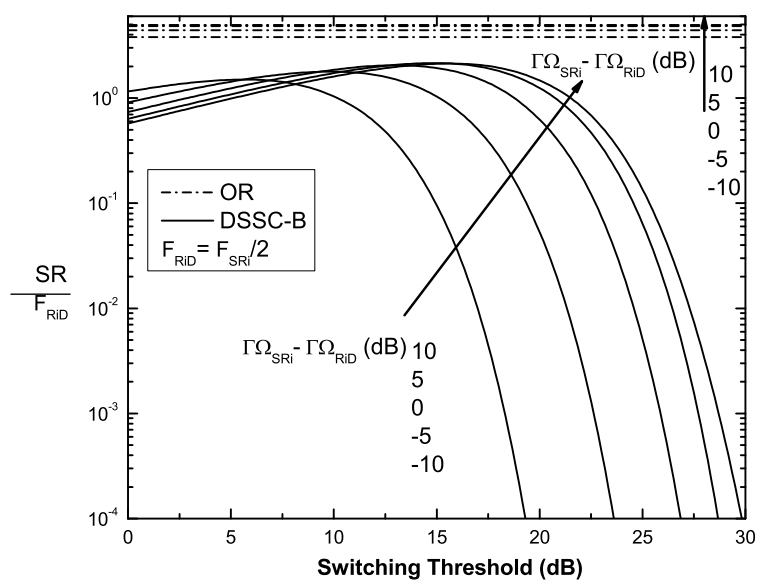

Fig. 2. Relay switching rates of OR and DSSC-B versus the switching threshold, $T$.

simpler implementation of selective cooperative relaying than OR.

\section{APPENDIX A}

\section{EVALUATION OF $f_{Z}(0)$}

The PDF of $Z(t)$ evaluated at zero reflects the probability that the absolute difference of the virtual fading gains of the $S-R_{1}-D$ and $S-R_{2}-D$ channels lies in the infinitesimal interval $[0, d z]$. Therefore, $f_{Z}(0)$ can be expressed as $f_{Z}(0)=$ $\int_{0}^{\infty} f_{a_{1}}(x) f_{a_{2}}(x) d x$, where $f_{a_{1}}(\cdot)$ and $f_{a_{2}}(\cdot)$ denote the PDFs of the processes $a_{1}(t)$ and $a_{2}(t)$, respectively. Using
(1), the CDF of $a_{i}(t), i \in\{1,2\}$, is expressed as

$$
\begin{aligned}
F_{a_{i}}(x) & =1-\int_{x}^{\infty} f_{S R_{i}}(\omega) d \omega \int_{x}^{\infty} f_{R_{i} D}(\omega) d \omega \\
& =1-\exp \left(-\frac{\Omega_{S R_{i}}+\Omega_{R_{i} D}}{\Omega_{S R_{i}} \Omega_{R_{i} D}} x^{2}\right)
\end{aligned}
$$

while the PDF of $a_{i}(t)$ is derived by differentiating (20), yielding

$$
f_{a_{i}}(x)=2 x \frac{\Omega_{S R_{i}}+\Omega_{R_{i} D}}{\Omega_{S R_{i}} \Omega_{R_{i} D}} e^{-\frac{\left(\Omega_{S R_{i}}+\Omega_{R_{i} D}\right) x^{2}}{\Omega_{S R_{i} \Omega_{R_{i}} D}}} .
$$

Note that the process $a_{i}(t)$ is also Rayleigh distributed; its average squared value is denoted by $\Omega_{i}$ and is given by $\Omega_{i}=$ $\Omega_{S R_{i}} \Omega_{R_{i} D} /\left(\Omega_{S R_{i}}+\Omega_{R_{i} D}\right)$.Then, $f_{Z}(0)$ is derived as the integral of the product of two Rayleigh distributions, yielding

$$
f_{Z}(0)=\frac{4}{\Omega_{1} \Omega_{2}} \int_{0}^{\infty} x^{2} e^{-\frac{\Omega_{1}+\Omega_{2}}{\Omega_{1} \Omega_{2}} x^{2}} d x .
$$

Using [12, eq. (3.321.5)], (22) yields (12).

\section{APPENDIX B}

$$
\text { DERIVATION OF } f_{\dot{Z}}(\cdot)
$$

The time derivative of $Z(t), Z(t)$, equals the difference of the time derivatives $\dot{a}_{1}(t)$ and $\dot{a}_{2}(t)$. Before proceeding in deriving $f_{\dot{z}}(\dot{z})$, we first obtain the PDF of $\dot{a}_{i}(t), i \in\{1,2\}$, as

$$
\begin{aligned}
f_{\dot{a}_{i}}(x)= & \operatorname{Pr}\left\{a_{S R_{i}} \leq a_{R_{i} D}\right\} f_{\dot{a}_{S R_{i}}}(x) \\
& +\operatorname{Pr}\left\{a_{S R_{i}}>a_{R_{i} D}\right\} f_{\dot{a}_{R_{i} D}}(x)
\end{aligned}
$$

where $f_{\dot{a}_{S R_{i}}}(\cdot)$ and $f_{\dot{a}_{R_{i} D}}(\cdot)$ denote the PDF of the time derivatives of $a_{S R_{i}}$ and $a_{R_{i} D}$, respectively. Given that $a_{S R_{i}}$ and $a_{R_{i} D}$ are Rayleigh distributed, $f_{\dot{a}_{S R_{i}}}(\cdot)$ and $f_{\dot{a}_{R_{i} D}}(\cdot)$ can be expressed as [9] $f_{\dot{a}_{S R_{i}}}(x)=\exp \left(-x^{2} / 2 \dot{\sigma}_{S R_{i}}^{2}\right) /\left(\sqrt{2 \pi} \dot{\sigma}_{S R_{i}}\right)$ and $f_{\dot{a}_{R_{i} D}}(x)=\exp \left(-x^{2} / 2 \dot{\sigma}_{R_{i} D}^{2}\right) /\left(\sqrt{2 \pi} \dot{\sigma}_{R_{i} D}\right)$, respectively; that is, $\dot{a}_{S R_{i}}$ and $\dot{a}_{R_{i} D}$ are zero-mean Gaussian random variables (RVs) with standard deviations $\dot{\sigma}_{S R_{i}}=\pi \mathcal{F}_{S R_{i}} \sqrt{\Omega_{S R_{i}}}$ and $\dot{\sigma}_{R_{i} D}=\pi \mathcal{F}_{R_{i} D} \sqrt{\Omega_{R_{i} D}}$, respectively. The steady-state probabilities $\operatorname{Pr}\left\{a_{S R_{i}} \leq a_{R_{i} D}\right\}$ and $\operatorname{Pr}\left\{a_{S R_{i}}>a_{R_{i} D}\right\}$ are given by

$$
\begin{aligned}
& \operatorname{Pr}\left\{a_{S R_{i}} \leq a_{R_{i} D}\right\}=\int_{0}^{\infty} f_{a_{S R_{i}}}(x)\left[1-F_{a_{R_{i} D}}(x)\right] d x \\
&=\frac{\Omega_{R_{i} D}}{\Omega_{S R_{i}}+\Omega_{R_{i} D}} \\
& \operatorname{Pr}\left\{a_{S R_{i}}>a_{R_{i} D}\right\}=\frac{\Omega_{S R_{i}}}{\Omega_{S R_{i}}+\Omega_{R_{i} D}} .
\end{aligned}
$$




$$
\begin{aligned}
f_{\dot{Z}}(x) & =\frac{\left(\Omega_{S R_{1}}+\Omega_{R_{1} D}\right)^{-1}}{\sqrt{2} \pi^{3 / 2}\left(\Omega_{S R_{2}}+\Omega_{R_{2} D}\right)}\left[\frac{\Omega_{S R_{1}} \Omega_{S R_{2}} e^{-\frac{x^{2}}{2 \pi^{2}\left(\Omega_{R_{1} D} \mathcal{F}_{R_{1} D}^{2}+\Omega_{R_{2} D} \mathcal{F}_{R_{2} D}^{2}\right)}}}{\sqrt{\Omega_{R_{1} D} \mathcal{F}_{R_{1} D}^{2}+\Omega_{R_{2} D} \mathcal{F}_{R_{2} D}^{2}}}+\frac{\Omega_{R_{1} D} \Omega_{S R_{2}} e^{-\frac{x^{2}}{2 \pi^{2}\left(\Omega_{S R_{1}} \mathcal{F}_{S R_{1}}^{2}+\Omega_{R_{2} D} \mathcal{F}_{R_{2} D}^{2}\right)}}}{\sqrt{\Omega_{S R_{1}} \mathcal{F}_{S R_{1}}^{2}+\Omega_{R_{2} D} \mathcal{F}_{R_{2} D}^{2}}}\right. \\
& \left.+\frac{\Omega_{R_{2} D} \Omega_{S R_{1}} e^{-\frac{x^{2}}{2 \pi^{2}\left(\Omega_{S R_{2}} \mathcal{F}_{S R_{2}}^{2}+\Omega_{R_{1} D} \mathcal{F}_{R_{1} D}^{2}\right)}}}{\sqrt{\Omega_{S R_{2}} \mathcal{F}_{S R_{2}}^{2}+\Omega_{R_{1} D} \mathcal{F}_{R_{1} D}^{2}}}+\frac{\Omega_{R_{1} D} \Omega_{R_{2} D} e^{-\frac{x^{2}\left(\Omega_{S R_{1}} \mathcal{F}_{S R_{1}}+\Omega_{S R_{2}} \mathcal{F}_{S R_{2}}^{2}\right)}{2 \pi_{2}}}}{\sqrt{\Omega_{S R_{1}} \mathcal{F}_{S R_{1}}^{2}+\Omega_{S R_{2}} \mathcal{F}_{S R_{2}}^{2}}}\right]
\end{aligned}
$$

The PDF of $\dot{a}_{i}(t)$ is therefore derived as

$$
\begin{aligned}
& f_{\dot{a}_{i}}(x)=\frac{\Omega_{R_{i} D} \mathcal{F}_{R_{i} D} e^{-\frac{x^{2}}{2 \pi^{2} \mathcal{F}_{S R_{i}}^{2} \Omega_{S R_{i}}}}}{\sqrt{2} \pi^{3 / 2}\left(\Omega_{S R_{i}}+\Omega_{R_{i} D}\right) \sqrt{\Omega_{S R_{i}}} \mathcal{F}_{S R_{i}} \mathcal{F}_{R_{i} D}} \\
& +\frac{\Omega_{S R_{i}} \mathcal{F}_{S R_{i}} e^{-\frac{x^{2}}{2 \pi^{2} \mathcal{F}_{R_{i} D^{2} R_{i} D}^{2}}}}{\sqrt{2} \pi^{3 / 2}\left(\Omega_{S R_{i}}+\Omega_{R_{i} D}\right) \sqrt{\Omega_{R_{i} D}} \mathcal{F}_{S R_{i}} \mathcal{F}_{R_{i} D}}
\end{aligned}
$$

where $i \in\{1,2\}$. Consequently, it follows from (9) that the PDF of the process $\dot{Z}(t)$ is given by [13, ch. 6] $f_{\dot{Z}}(x)=$ $\int_{-\infty}^{\infty} f_{\dot{a}_{1}}(y+x) f_{\dot{a}_{2}}(y) d y$. Hence, making use of the integral $[12$, Eq. (3.323.2)]

$$
\int_{-\infty}^{\infty} e^{-\frac{(y+x)^{2}}{\alpha_{1}}} e^{-\frac{x^{2}}{\alpha_{2}}} d y=\sqrt{\pi \frac{\alpha_{1} \alpha_{2}}{\alpha_{1}+\alpha_{2}}} e^{-\frac{x^{2}}{\alpha_{1}+\alpha_{2}}}
$$

we derive the PDF of $\dot{Z}(t), f_{\dot{Z}}(\cdot)$, as shown in (26) at the top of this page.

Having an expression for $f_{\dot{r}}(\cdot)$, the last term in (11b) is derived using [12, Eq. (3.321.4)] as shown in (13).

\section{APPENDIX C}

\section{Steady-State Relay Activation Probabilities of DSSC-B}

Considering that relay switchings in DSSC-B are determined in exactly the same way as branch switchings in SSC$\mathrm{B}$, the steady-state relay activation probabilites for DSSC-B are derived through the Markov states of SSC-B given in [8]. Specifically, the Markov chain of DSSC-B yields six states, as follows. State 1 corresponds to the case where " $R_{1}$ is active and the overal SNR down-crosses T"; state 2 corresponds to " $R_{1}$ is active and the overall SNR is below $T$ "; state 3 corresponds to " $R_{1}$ is active and the overall SNR is greater than T"; states 4, 5, 6 refer to the case where $R_{2}$ is active, and are defined analogous to $1,2,3$, respectively. The stationary probabilities, $\pi_{j}^{D S S C-B}, j \in\{1, \ldots, 6\}$, of the above Markov states are taken from $[8$, Eq. (21)], yielding

$$
\begin{aligned}
& \pi_{1}^{D S S C-B}=\frac{\left[1-q_{1}\right] q_{1}\left[1-q_{2}\right] q_{2}}{\left[q_{1}+q_{2}\right]\left[1+2 q_{1} q_{2}\right]-\left[q_{1}+q_{2}\right]^{2}-2 q_{1}^{2} q_{2}^{2}} \\
& \pi_{2}^{D S S C-B}=\frac{q_{1}^{2} q_{2}\left[1-q_{2}\right]}{\left[q_{1}+q_{2}\right]\left[1+2 q_{1} q_{2}\right]-\left[q_{1}+q_{2}\right]^{2}-2 q_{1}^{2} q_{2}^{2}}
\end{aligned}
$$

$$
\begin{aligned}
& \pi_{3}^{D S S C-B}=\frac{\left[1-q_{1}\right]^{2}\left[1-q_{2}\right] q_{2}}{\left[q_{1}+q_{2}\right]\left[1+2 q_{1} q_{2}\right]-\left[q_{1}+q_{2}\right]^{2}-2 q_{1}^{2} q_{2}^{2}} \\
& \pi_{4}^{D S S C-B}=\pi_{1}^{D S S C-B} \\
& \pi_{5}^{D S S C-B}=\frac{q_{2}^{2} q_{1}\left[1-q_{1}\right]}{\left[q_{1}+q_{2}\right]\left[1+2 q_{1} q_{2}\right]-\left[q_{1}+q_{2}\right]^{2}-2 q_{1}^{2} q_{2}^{2}} \\
& \pi_{6}^{D S S C-B}=\pi_{1}^{D S S C-B}
\end{aligned}
$$

where $q_{1}=F_{a_{1}^{2}}(T / \Gamma) ; q_{2}=F_{a_{2}^{2}}(T / \Gamma)$. Considering that $R_{1}$ is active in states $1,2,3$, while $R_{2}$ in states $4,5,6$, the steadystate relay activation probabilities of DSSC-B are derived as $\rho_{1}^{D S S C}=\sum_{j=1}^{3} \pi_{j}^{D S S C-B} ; \rho_{2}^{D S S C}=\sum_{j=4}^{6} \pi_{j}^{D S S C-B}$, yielding (17) and (18).

\section{REFERENCES}

[1] J. N. Laneman, D. N. C. Tse, and G. W. Wornell, "Cooperative diversity in wireless networks: Efficient protocols and outage behavior," IEEE Trans. Inform. Theory, vol. 50, pp. 3062-3080, Dec. 2004.

[2] Y. Zhao, R. Adve, and T. J. Lim, "Improving amplify-and-forward relay networks: optimal power allocation versus selection," in Proc. IEEE Intern. Symp. Inform. Theory (ISIT 06), Seattle, WA, Jul. 2006.

[3] A. Bletsas, A. Khisti, D. P. Reed, and A. Lippman, "A simple cooperative diversity method based on network path selection," IEEE J. Selec. Areas Commun., vol. 24, pp. 659-672, Mar. 2006.

[4] D. S. Michalopoulos and G. K. Karagiannidis, "Performance analysis of single relay selection in Rayleigh fading," IEEE Trans. Wireless Commun., vol. 7, pp. 3718-3724, Oct. 2008 .

[5] R. Tannious and A. Nosratinia, "Spectrally efficient relay selection protocols in wireless networks," in Proc IEEE Intern. Conf. on Acoustics, Speech and Signal Processing (ICASSP), Las Vegas, NV, Mar. 2008.

[6] D. S. Michalopoulos and G. K. Karagiannidis, "Two relay distributed switch and stay combining (DSSC)," IEEE Trans. Commun., vol. 56, pp. 1790-1794, Nov. 2008.

[7] S. Ikki and M. H. Ahmed, "Performance analysis of cooperative diversity wireless networks over Nakagami- $m$ fading channel," IEEE Communications Letters, vol. 11, pp. 334-336, Apr. 2007.

[8] H.-C. Yang and M.-S. Alouini, "Markov chains and performance comparison of switched diversity systems," IEEE Trans. Commun., vol. 52, pp. 1113-1125, Jul. 2004.

[9] S. Rice, "Statistical properties of a sine wave plus noise," Bell Syst. Tech. J., vol. 27, pp. 109-157, Jan. 1948.

[10] N. C. Beaulieu, "Switching rates of dual selection diversity and dual switch-and-stay diversity," IEEE Trans. Commun., vol. 56, pp. 14091413, Sep. 2008.

[11] F. Sivrikaya and B. Yener, "Time synchronization in sensor networks: A survey," IEEE Network J., vol. 18, pp. 45-50, Jul. 2004.

[12] I. S. Gradshteyn and I. M. Ryzhik, Table of Integrals, Series, and Products, 6th ed. New York: Academic, 2000.

[13] A. Papoulis, Probability, Random Variables, and Stochastic Procceses, 3rd ed. McGraw-Hill, 1991. 